\title{
Leptin and regulatory T-lymphocytes in idiopathic pulmonary arterial hypertension
}

\author{
Alice Huertas, Ly Tu, Natalia Gambaryan, Barbara Girerd, Frédéric Perros, \\ David Montani, Dominique Fabre, Elie Fadel, Saadia Eddahibi, \\ Sylvia Cohen-Kaminsky, Christophe Guignabert and Marc Humbert
}

\begin{abstract}
Immune mechanisms and autoimmunity seem to play a significant role in idiopathic pulmonary arterial hypertension (IPAH) pathogenesis and/or progression, but the pathophysiology is still unclear. Recent evidence has demonstrated a detrimental involvement of leptin in promoting various autoimmune diseases by controlling regulatory T-lymphocytes. Despite this knowledge, the role of leptin in IPAH is currently unknown. We hypothesised that leptin, synthesised by dysfunctional pulmonary endothelium, might play a role in the immunopathogenesis of IPAH by regulating circulating regulatory $\mathrm{T}$-lymphocytes function.

First, we collected serum and regulatory T-lymphocytes from controls, and IPAH and sclerodermaassociated pulmonary arterial hypertension (SSc-PAH) patients; secondly, we recovered tissue samples and cultured endothelial cells after either surgery or transplantation in controls and IPAH patients, respectively.

Our findings indicate that serum leptin was higher in IPAH and SSc-PAH patients than controls. Circulating regulatory T-lymphocyte numbers were comparable in all groups, and the percentage of those expressing leptin receptor was higher in IPAH and SSc-PAH compared with controls, whereas their function was reduced in IPAH and SSC-PAH patients compared with controls, in a leptin-dependent manner. Furthermore, endothelial cells from IPAH patients synthesised more leptin than controls.

Our data suggest that endothelial-derived leptin may play a role in the immunopathogenesis of IPAH.
\end{abstract}

KEYWORDS: Dysimmunity, endothelial dysfunction, leptin, pulmonary arterial hypertension, regulatory T-lymphocytes

diopathic pulmonary arterial hypertension (IPAH) corresponds to pre-capillary pulmonary hypertension in which there is neither a family history of the disease nor an identified risk factor and is defined as a resting mean pulmonary arterial pressures $\geqslant 25 \mathrm{mmHg}$ with a normal pulmonary artery wedge pressure $\leqslant 15 \mathrm{mmHg}$ [1]. Although the pathophysiology of IPAH has been extensively studied in the past few decades and several new pathways have been identified, the aetiology of this disease is still not clearly understood. It is now well established that inflammation plays an important role in IPAH [2-6], and increasing data also support the hypothesis that immunological disorders could be present in IPAH patients: circulating autoantibodies have been detected $[7,8]$ and recent evidence indicates that regulatory T-cells (Tregs) could play a role in pulmonary arterial hypertension (PAH)
[9]. Despite these findings, little is known about the exact role of inflammation and dysimmunity in the development of IPAH and it remains unclear how immune mechanisms contribute to the pathogenesis of IPAH.

Recent evidence has demonstrated a detrimental involvement of leptin, a cytokine-like hormone mainly secreted by adipocytes, in promoting the pathogenesis of various autoimmune diseases $[10,11]$. Although leptin, the product of the obese $(o b)$ human gene, has been discovered as the appetite regulator, it regulates a wide range of physiological functions [10-13]. Interestingly, it has also been shown that leptin can promote chronic autoimmune disorders by regulating Treg onset and/or function [14]. Intriguingly, little is known about the pulmonary effects of leptin, and, in particular, its inflammatory and/or immunological role in IPAH.
AFFILIATIONS

Université Paris-Sud, Faculté de Médecine, Le Kremlin-Bicêtre; Centre National de Référence de l'Hypertension Pulmonaire Sévère, Service de Pneumologie et Réanimation Respiratoire, Hôpital Bicêtre, AP-HP, Le Kremlin-Bicêtre; and INSERM U999 Hypertension Artérielle Pulmonaire, Physiopathologie et Innovation Thérapeutique, LabEX LERMIT Laboratory of Excellence in Research on Medication and Innovative Therapeutics (LERMIT), Centre Chirurgical Marie Lannelongue, Le Plessis-Robinson, France.

\section{CORRESPONDENCE}

M. Humbert

Service de Pneumologie et

Réanimation Respiratoire

Centre National de Référence de

l'Hypertension Pulmonaire Sévère

Hôpital Bicêtre

Hôpitaux Universitaires Paris-Sud Assistance Publique Hôpitaux de

Paris

78 rue du Général Leclerc

94270

Le Kremlin-Bicêtre

France

E-mail: marc.humbert@bct.aphp.fr

Received:

Sept 142011

Accepted after revision:

Feb 062012

First published online:

Feb 232012 
Tregs are T-lymphocytes known to dampen autoreactive responses and are able to delay the onset and progression of autoimmune disorders: reduced frequency of Tregs and/or defective suppressor function have been observed in these diseases $[14,15]$. So far, little is known about the role of leptin in the lung and, in particular, about its effects on the pulmonary blood vessels.

IPAH is characterised by small-sized pulmonary arteries/ arterioles in which there is intimal hyperplasia with medial and adventitial hypertrophy, hyperplasia and fibrosis. Cells from the vessel wall are known to play an important role in the pathogenesis of IPAH. In particular, pulmonary endothelial cells (P-ECs) represent a critical cell type. Endothelial dysfunction is characterised by an altered vasoconstriction/vasodilatation balance and disorganised pro-proliferative and apoptoticresistant phenotype that can lead to the formation of plexiform lesions mostly described in IPAH patients [16].

We hypothesised that leptin, synthesised by dysfunctional PECs, might play a role in the immunopathology of IPAH by regulating circulating Treg function. We addressed this issue using freshly recovered circulating Tregs from IPAH patients. Our findings indicate that Treg function is reduced in IPAH patients compared with controls in a leptin-dependent manner. Furthermore, using cultured cells recovered after lung transplantation in IPAH patients, we demonstrated that there is a major increase in leptin synthesis from IPAH P-EC compared with controls. Taken together, our data suggest that leptin could derive from endothelial cells and may play a role in the immunopathogenesis and/or progression of IPAH.

\section{METHODS}

\section{Subjects}

Blood samples were collected in patients with IPAH and in scleroderma (SSc)-associated PAH during usual follow-up and in control subjects (table 1). All patients were treated with PAH-specific treatments. Inclusion criteria were aged $>18$ yrs and PAH diagnosis confirmed by right heart catheterisation with a stable clinical and haemodynamic status for the last 3 months. Exclusion criteria were a heritable form of PAH, anaemia, thyroid dysfunction, diabetes, metabolic syndrome, and immunosuppressive or corticosteroid therapies in the last 6 months for SSc-PAH. Characteristics at diagnosis and follow-up were stored in the Registry of the French Network of Pulmonary Hypertension set up in agreement with French bioethics laws (Commission Nationale de l'Informatique et des Libertés), and all patients gave their written informed consent.

\section{Leptin, leptin receptor and pro-inflammatory measurements} Serum samples were collected and stored at $-80^{\circ} \mathrm{C}$. For the measurement of leptin, C-reactive protein (CRP), tumour necrosis factor (TNF)- $\alpha$, monocyte chemoattractant protein (MCP)-1, interleukin (IL)-6 and -1 $\beta$, human AlphaLISA (Perkin Elmer, Waltham, MA, USA) or ELISAs (R\&D Systems, Minneapolis, MN, USA) kits were used accordingly to manufacturer's instructions. Circulating soluble leptin receptor (sObR) levels were measured using ELISAs (as recommended by the manufacturer, R\&D Systems, Minneapolis, MN, USA).

\begin{tabular}{|c|c|c|c|}
\hline \multirow[t]{2}{*}{ TABLE 1} & \multirow[b]{2}{*}{ Controls } & modynamic & \multirow[b]{2}{*}{ SSc-PAH } \\
\hline & & IPAH & \\
\hline Subjects $\mathrm{n}$ & 20 & 25 & 11 \\
\hline Females/males & $12 / 8(0.66)$ & $20 / 5(0.25)$ & 9/2 (0.22) \\
\hline Age yrs & $38 \pm 3^{*}$ & $52 \pm 3$ & $68 \pm 3^{\#}$ \\
\hline BMI $\mathrm{kg} \cdot \mathrm{m}^{-2}$ & $25 \pm 1$ & $26 \pm 1$ & $27 \pm 1$ \\
\hline NYHA class & NA & $\|-\| I$ & II-IV \\
\hline Mean Ppa mmHg & NA & $45 \pm 3$ & $41 \pm 4$ \\
\hline Ppcw mmHg & NA & $8 \pm 1$ & $9 \pm 1$ \\
\hline PVR Wood units & NA & $7 \pm 1$ & $7 \pm 1$ \\
\hline Cardiac output L. $\min ^{-1}$ & NA & $5.8 \pm 0.3$ & $5.5 \pm 0.6$ \\
\hline Cardiac index L $\min ^{-1} \cdot \mathrm{m}^{-2}$ & NA & $3.0 \pm 0.2$ & $3.0 \pm 0.3$ \\
\hline 6MWT distance m & NA & $459 \pm 21$ & $314 \pm 44^{\#}$ \\
\hline$B N P \mathbf{n g} \cdot \mathrm{mL}^{-1}$ & NA & $103 \pm 28$ & $250 \pm 92$ \\
\hline CRP $\mathrm{mg} \cdot \mathrm{L}^{-1}$ & $0.05 \pm 0.003$ & $0.05 \pm 0.002$ & $0.04 \pm 0.005$ \\
\hline $\mathrm{TNF}-\alpha \mathrm{pg} \cdot \mathrm{mL}^{-1}$ & $0.5 \pm 0.4$ & $4.2 \pm 2.0$ & $2.2 \pm 2$ \\
\hline $\mathrm{MCP}-1 \mathrm{pg} \cdot \mathrm{mL}^{-1}$ & $273 \pm 19^{*}$ & $682 \pm 158$ & $460 \pm 42$ \\
\hline $\mathrm{IL}-6 \mathrm{pg} \cdot \mathrm{mL}^{-1}$ & $1 \pm 0.4^{*}$ & $25 \pm 9$ & $8 \pm 2$ \\
\hline $\mathrm{IL}-1 \beta \mathrm{pg} \cdot \mathrm{mL}^{-1}$ & $0.01 \pm 0.01^{*}$ & $32 \pm 8$ & $17 \pm 12$ \\
\hline PAH-specific treatment $n$ & NA & & \\
\hline ERA & & 11 & 3 \\
\hline PDE-5i & & 0 & 1 \\
\hline ERA+PDE-5i & & 8 & 4 \\
\hline $\mathrm{ERA}+\mathrm{PGl}_{2}$ & & 2 & 0 \\
\hline PDE-5i+PGl 2 & & 2 & 0 \\
\hline ERA+PDE-5i+PGl 2 & & 2 & 3 \\
\hline No treatment & & 0 & 0 \\
\hline
\end{tabular}

Data are presented as $n / n$ (\%) or mean $\pm S D$, unless otherwise stated. IPAH: idiopathic pulmonary arterial hypertension; SSc-PAH: scleroderma-associated pulmonary arterial hypertension; BMI: body mass index; NYHA: New York Heart Association; Ppa: pulmonary artery pressure; Ppcw: pulmonary capillary wedge pressure; PVR: pulmonary vascular resistance; $6 \mathrm{MWT}$ : 6-min walk test; $B N P$ : brain natriuretic peptide; CRP: C-reactive protein; TNF: tumour necrosis factor; MCP: monocyte chemoattractant protein; IL: interleukin; ERA: endothelin receptor antagonist; PDE-5i: phosphodiesterase type-5 inhibitor; $\mathrm{PGI}_{2}$ : prostacyclin (prostaglandin $\mathrm{I}_{2}$ ); NA: not applicable. *: $\mathrm{p}<0.05$ between controls versus IPAH and versus SSc-PAH patients; ${ }^{*}: \mathrm{p}<0.05$ between IPAH and SSc-PAH patients.

\section{Flow cytometry analysis}

After blood samples were taken, peripheral blood mononuclear cells (PBMCs) from IPAH and SSc-PAH patients, and controls were obtained by standard Ficoll gradient centrifugation. The cells were carefully washed with PBS and resuspended in a staining buffer containing 10\% human serum. The cells were then fluorescently labelled with the following monoclonal antibodies: anti-CD4 conjugated with either Alexa 488 (Becton Dickinson, Franklin Lakes, NJ, USA) or Pacific Blue (BioLegend, San Diego, CA, USA); anti-CD25 conjugated with Alexa 647 (BioLegend); anti-CD127 conjugated with peridinin-chlorophyll protein-Cy5.5 (Becton Dickinson); and anti-FoxP3 conjugated with Pacific blue (BioLegend), under either nonpermeabilised or permeabilised conditions (IntraPrep, following manufacturer's instructions, Beckman Coulter, Brea, CA, USA) to enable either surface or intracellular staining, respectively. Flow cytometry gating conditions and the mean fluorescence intensity were set 
and normalised, respectively, against isotype- and fluorophorematched nonimmune immunoglobulin G.

It is now well known that CD127 expression inversely correlates with FoxP3 activation [17]. To test whether Treg analysis and cell count were similar using these different markers, we randomly selected five subjects from each group and compared the two "Treg phenotypes", i.e. $\mathrm{CD}^{+} \mathrm{CD} 25^{+} \mathrm{CD} 127^{\text {low }} \mathrm{FoxP}^{+}$ and $\mathrm{CD} 44^{+} \mathrm{CD} 25^{+} \mathrm{CD} 127^{\text {low }}$ (the subgroup characteristics are presented as online supplementary data and results are shown in figures 1-4). The cell count was similar for each of these two different surface markers (fig. $4 \mathrm{c}$ and table 2). Based on these preliminary tests and to preserve the intracellular space, we avoided PBMCs and defined Treg as $\mathrm{CD} 4^{+} \mathrm{CD} 25^{+} \mathrm{CD} 127^{\text {low }}$ (fig. 3a). Treg cell count was expressed as the percentage of total $\mathrm{CD} 4^{+}$cells.

In order to evaluate the ObR expression, PBMCs were also stained under nonpermeabilised conditions with a monoclonal antibody against ObR fluorescently conjugated with phycoerythrin (R\&D Systems). Tregs expressing ObR were defined as $\mathrm{CD} 4^{+} \mathrm{CD} 25^{+} \mathrm{CD} 127^{\text {low }} \mathrm{ObR}{ }^{+}$and were quantified as the percentage of $\mathrm{CD} 4^{+} \mathrm{CD} 25^{+} \mathrm{CD} 127^{\text {low }}$ cells.
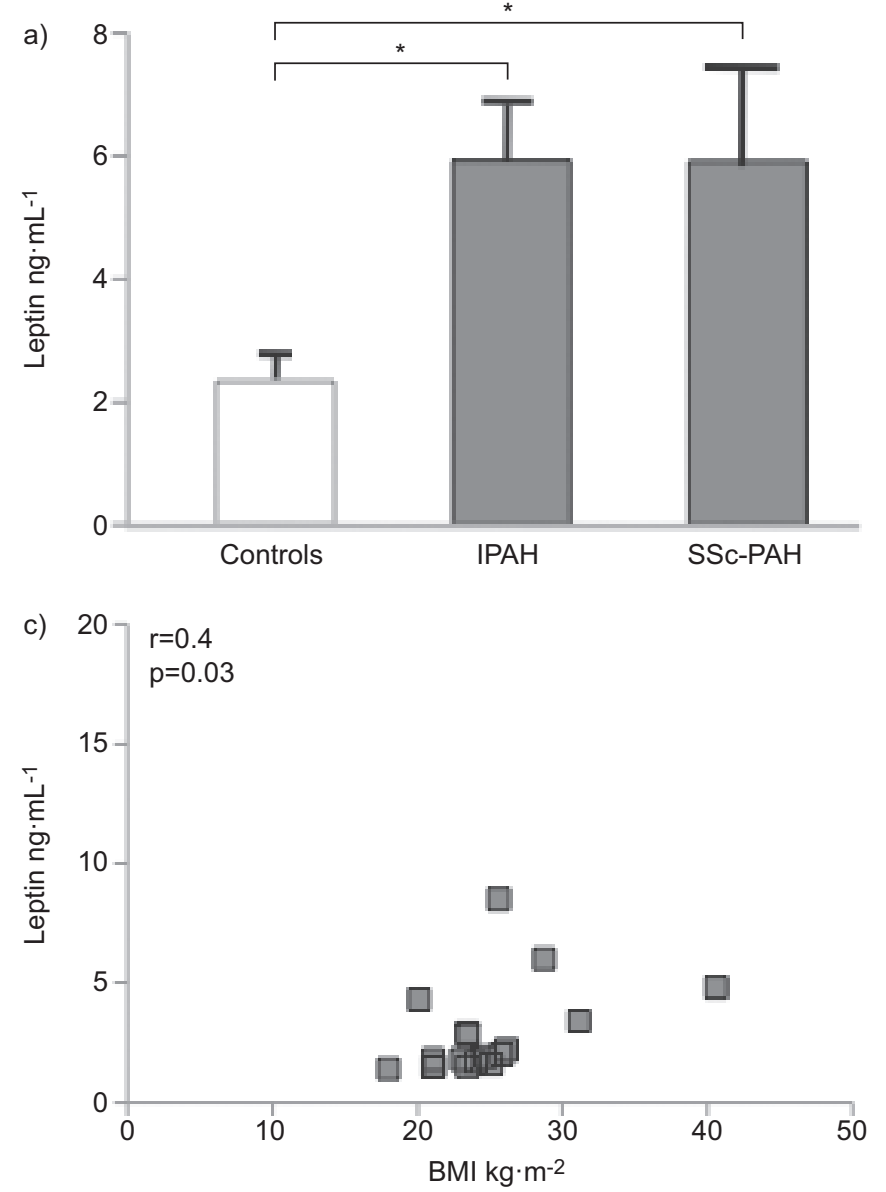

To determine the functional status of the Tregs expressing $\mathrm{ObR}$, we stained them under permeabilised conditions with a monoclonal antibody against phosphorylated signal transducer and activator of transcription 3 (pSTAT3) conjugated with Alexa 488 (Cell Signaling Technology, Danvers, MA, USA).

To assess the effect of leptin on Treg functional status, we stimulated freshly isolated PBMCs from healthy controls with recombinant human leptin at a concentration of $250 \mathrm{ng} \cdot \mathrm{mL}^{-1}$ for 5 min (R\&D Systems, Minneapolis, MN, USA) [18]. Then, we stained and analysed the Tregs and pSTAT3 as described above.

Flow cytometry data were acquired with a flow cytometer (MACSQuant, Myltenyi Biotec, Bergisch Gladbach, Germany) and analysed by FlowJo software program (Tree Star, Inc., Ashland, OR, USA).

\section{Human pulmonary tissues and immunohistostaining}

Lung specimens were obtained at the time of lung transplantation from patients with IPAH $(n=10)$, at the Marie Lannelongue Hospital, Le Plessis-Robinson, France. Control lung specimens were obtained from patients without any evidence of pulmonary vascular disease who underwent lobectomy or pneumonectomy
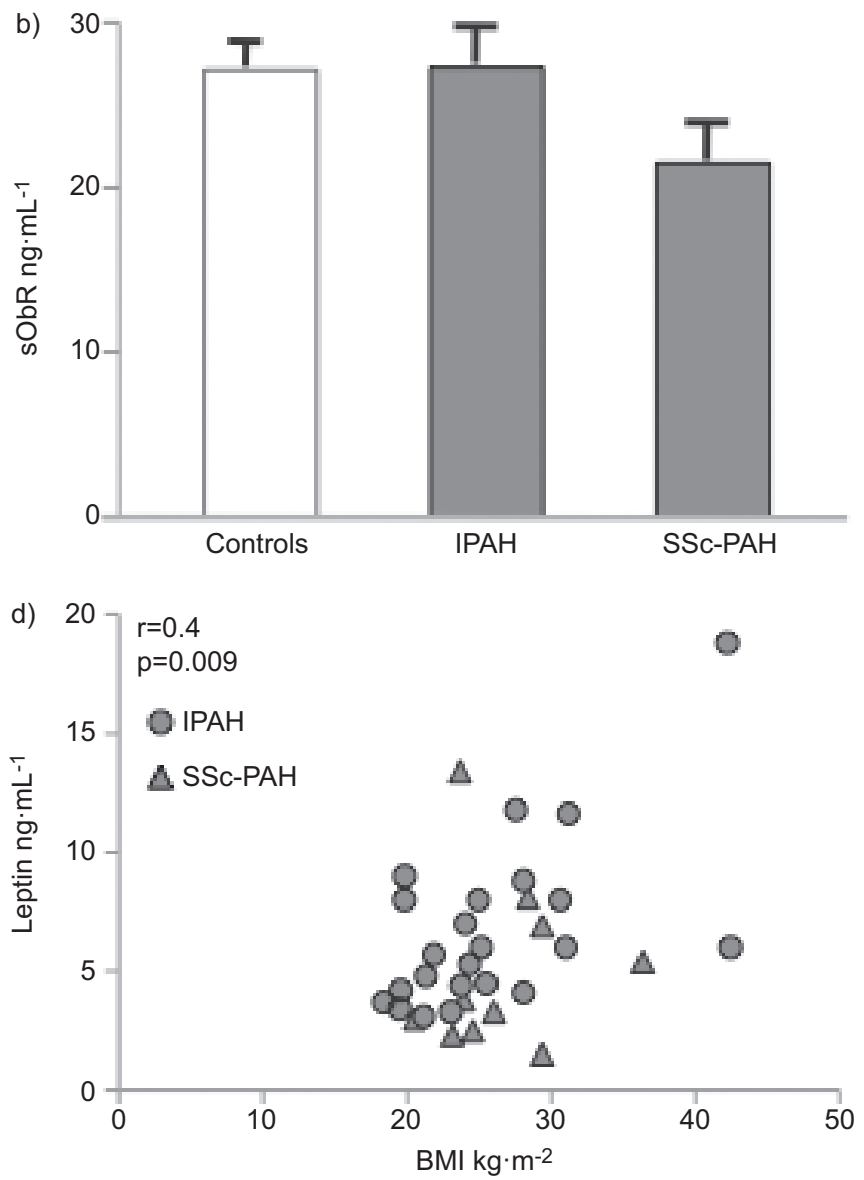

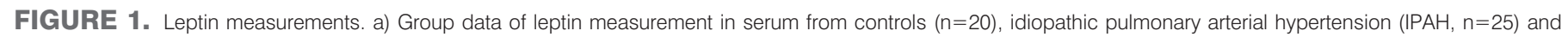

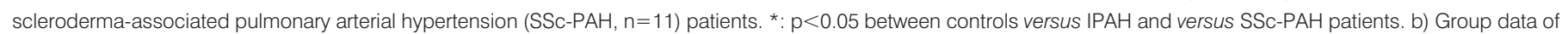

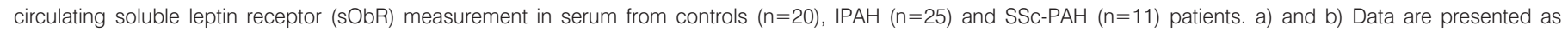
mean \pm SEM. Correlation between $\mathrm{c}$ ) serum leptin and body mass index (BMI) in controls and d) serum leptin and BMI in patients considered as a unique group. 

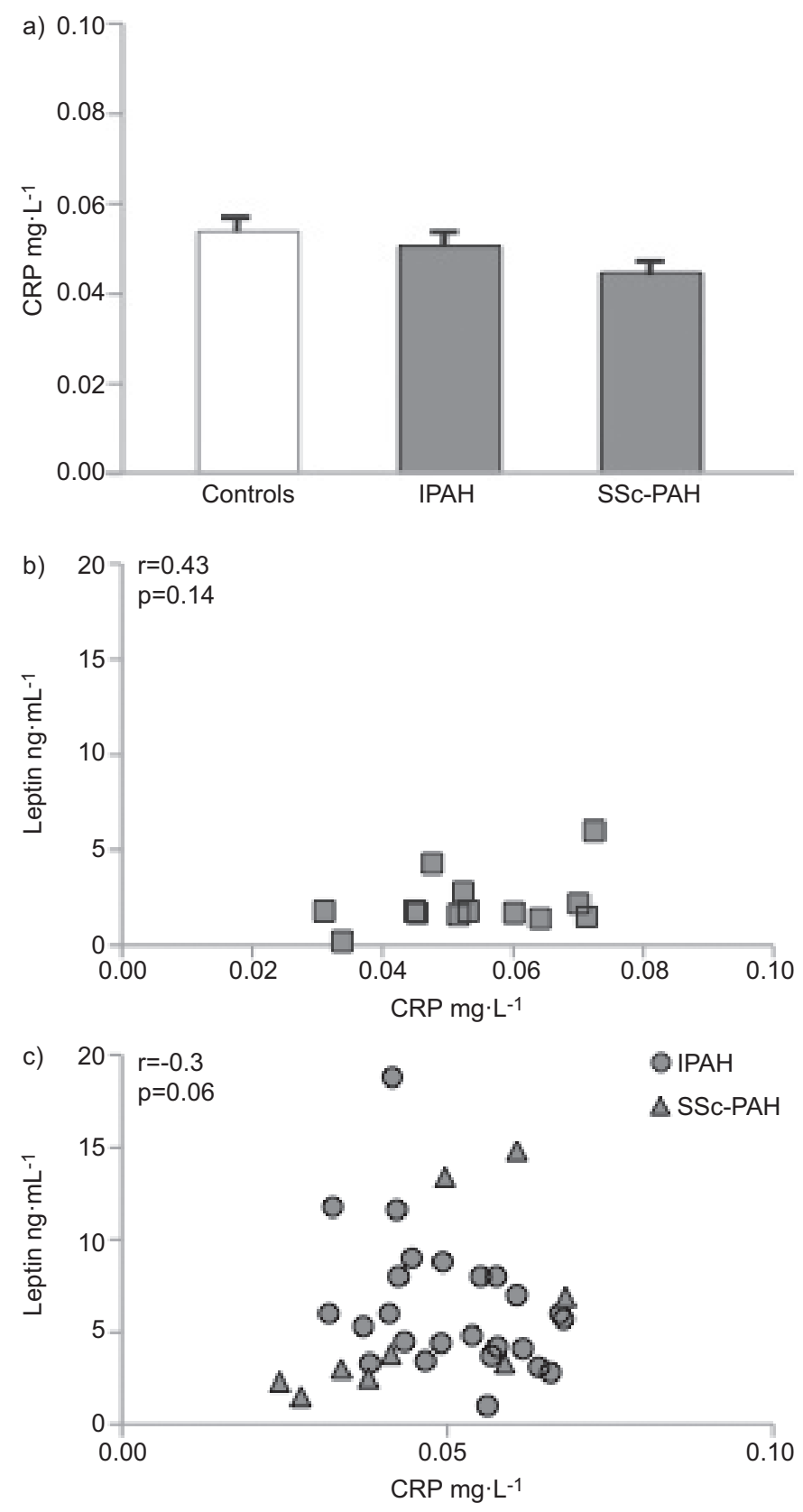

FIGURE 2. C-reactive protein (CRP) measurements. a) Group data of CRP measurement in serum from controls $(n=20)$, idiopathic pulmonary arterial hypertension (IPAH, $\mathrm{n}=25$ ) and scleroderma-associated pulmonary arterial hypertension (SSc-PAH, $\mathrm{n}=11$ ) patients. Data are presented as mean \pm SEM. Correlation between b) CRP and serum leptin in controls and c) CRP and serum leptin in patients considered as a unique group.

for localised lung cancer $(n=15)$. Lung specimens were fixed in $4 \%$ paraformaldehyde and embedded in paraffin. $5-\mu \mathrm{m}$ sections were dewaxed and rehydrated progressively. Citrate buffer ( $\mathrm{pH}$ 6) was used for antigen retrieval, and endogenous peroxidase was quenched with hydrogen peroxide. Sections were blocked with $5 \%$ bovine serum albumin and incubated overnight with the following antibodies: polyclonal antibody against leptin (dilution 1/100; Santa Cruz Biotechnologies, Santa Cruz, CA, USA) and monoclonal antibody against leptin receptor (dilution 1/50; R\&D Systems). Biotin-streptavidinperoxidase systems, 3,3'-diaminobenzidine (DAB) substrate was used for relevation (Universal LSAB HRP Kit; Dako, Trappes, France). Nuclei were counterstained with haematoxylin and mounted. Controls used for these antibodies included omission of the primary antibody and substitution of the primary antibody by an isotype control.

\section{Endothelial cell isolation and leptin measurement}

Human microvascular P-ECs isolated from lung tissue fragments using immunomagnetic purification were cultured as previously described [19]. To quantify leptin in conditioned media, the isolated cells were seeded on six-well plates at a density of $1 \times 10^{5}$ cells per well. After $24 \mathrm{~h}$, cells were washed twice with PBS then incubated for $24 \mathrm{~h}$ in serum-free MCDB131 (without the addition of growth factors). Leptin was measured in conditioned media using ELISAs (as recommended by the manufacturer; R\&D Systems).

\section{Statistical analysis}

Results are expressed as mean \pm SEM. A $p<0.05$ level of statistical significance was used for all analyses. The Shapiro-Wilk test was used to ensure that data had a normal distribution. All betweengroups comparisons were assessed using one-way ANOVA; post hoc analysis of significant variables was performed using Tukey's test with all pairwise multiple comparisons. Differences between two selected groups (controls and IPAH) were compared using unpaired t-test. Pearson correlations were used to establish associations between the dependent variables (i.e. leptin and ObR expression on Tregs) and relevant independent variables. All statistical procedures were carried out using GraphPad Prism version 5.0 (GraphPad Software Inc., San Diego, CA, USA).

\section{RESULTS \\ Leptin and pro-inflammatory cytokines serum concentration}

To investigate the role of leptin in $\mathrm{PAH}$ pathogenesis, we first quantified serum leptin concentration and found that both IPAH and SSc-PAH patients had a significantly higher leptinaemia than controls. No difference was found between IPAH and SScPAH (fig. 1a and table 2).

In order to compare the relative free leptin index and the leptin bioavailability in these three groups of subjects, we measured sObR levels. We found a similar level in the three groups without any statistically significant difference (fig. $1 \mathrm{~b}$ and table 2). These data indicate that circulating active leptin levels are increased in both IPAH and SSc-PAH. Correlations between serum leptin levels and body mass index (BMI) in controls and $\mathrm{PAH}$ patients are shown in figures $1 \mathrm{c}$ and $\mathrm{d}$. Furthermore, no correlation was found between circulating leptin levels and disease severity expressed as haemodynamic and/or functional parameters. As leptin has been shown to act as a key player in inflammatory conditions, we tested whether serum leptin concentration in PAH correlated with serum pro-inflammatory cytokines. CRP and TNF- $\alpha$ concentrations were normal in all groups, without any statistically significant difference among the groups (fig. $2 \mathrm{a}$ and table 1). MCP-1 and IL-6 and $-1 \beta$ were significantly increased in IPAH and SSc-PAH patients compared with controls (table 1). Furthermore, there was no correlation between the pro-inflammatory cytokines and leptin levels (figs $2 b$ and $c$ ). 
a)
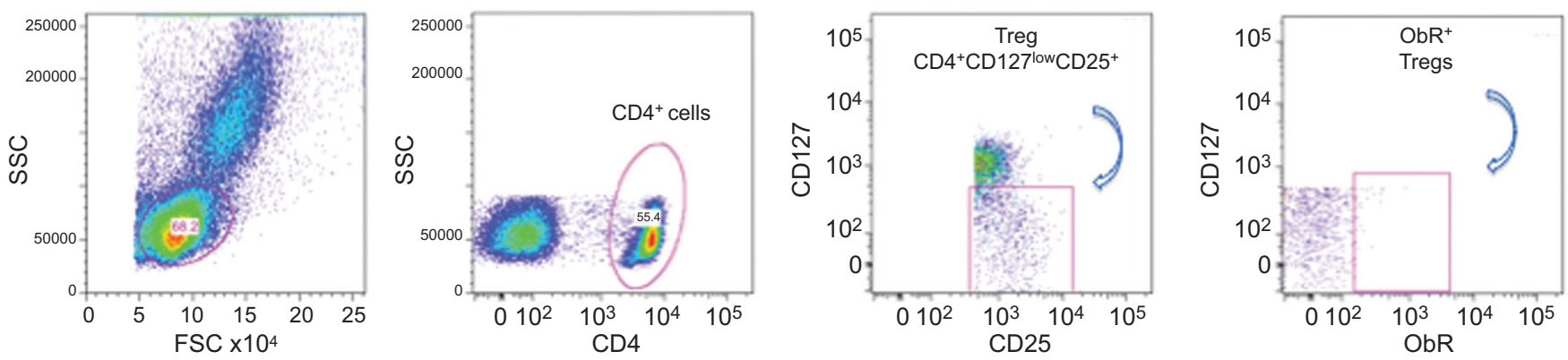

b)
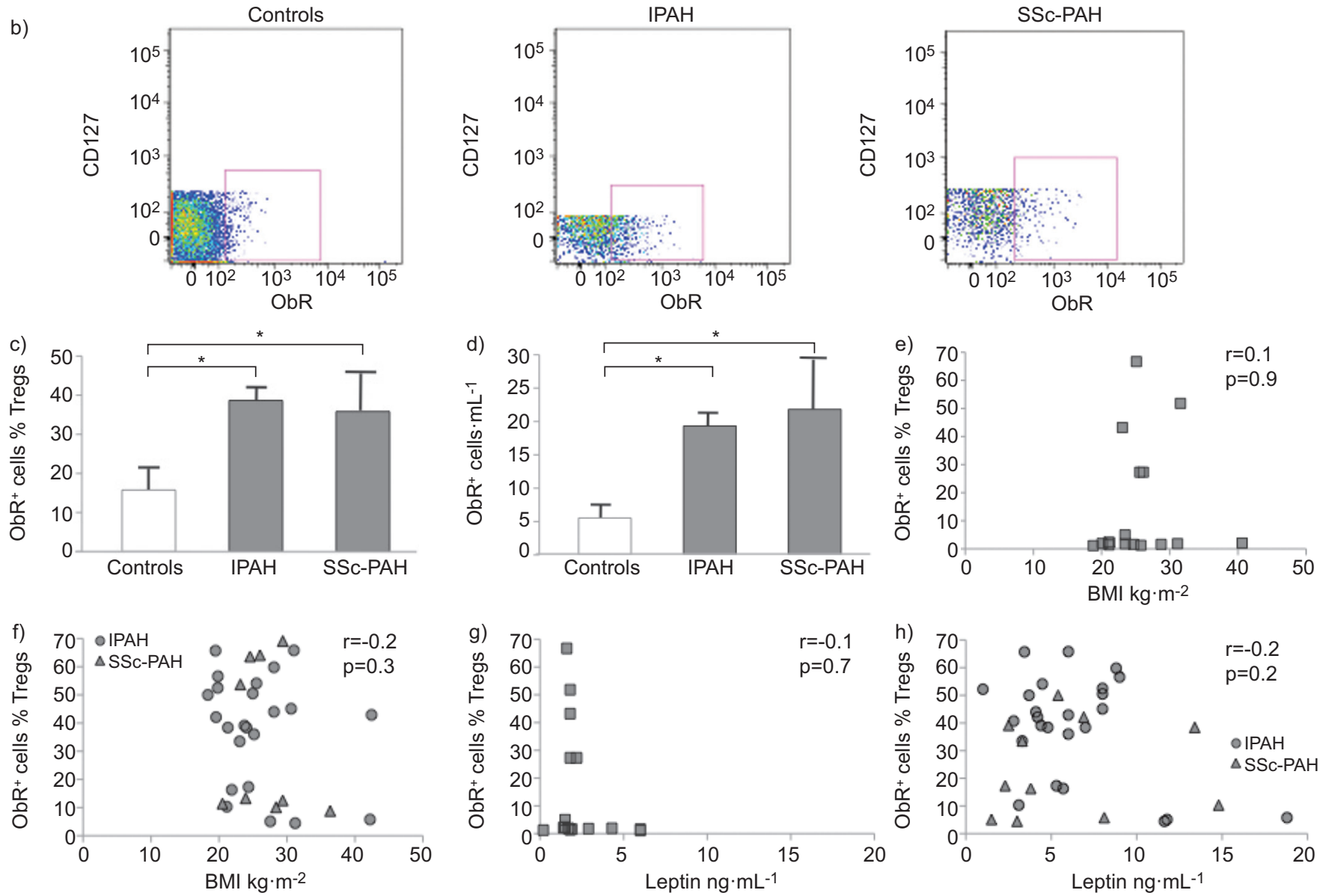

FIGURE 3. Leptin receptor (ObR) expression. a) Single fluorescence-activated cell sorter (FACS) dot plots representing an example of phenotype strategy by using surface stainings analysed by flow cytometry in a control subject. b) Single FACS dot plot representing ObR ${ }^{+}$regulatory T-lymphocytes (Tregs) in control subjects and idiopathic pulmonary arterial hypertension (IPAH) and scleroderma-associated pulmonary arterial hypertension (SSc-PAH) patients. SSC: side scatter; FSC: forward scatter. Group data of ObR expression on circulating Tregs, expressed as $\mathrm{c}$ ) a percentage of the total Treg population and d) absolute numbers, in control subjects ( $\mathrm{n}=20$ ), and IPAH $(n=25)$ and SSc-PAH $(n=11)$ patients. Data are presented as mean \pm SEM. ${ }^{*}: p<0.05$ between controls versus IPAH and versus SSc-PAH patients. Correlation between ObR ${ }^{+}$ Tregs and body mass index (BMl) in e) controls. and f) patients considered as a unique. Correlation between ObR ${ }^{+}$Tregs and serum leptin in g) controls and h) patients considered as a unique group.

These data indicate that in PAH patients, in IPAH and in SSc$\mathrm{PAH}$, serum leptin levels are increased independently of proinflammatory cytokines.

\section{ObR expression on Treg cell membrane}

As our findings indicated a possible role of leptin in $\mathrm{PAH}$ pathogenesis, we analysed the expression of its receptor ObR on Tregs. After withdrawing peripheral venous blood samples from controls and PAH patients, we first selected Tregs among the fluorescently tagged PBMCs by flow cytometry analysis.

We selected Tregs and analysed the expression of ObR on their membranes (fig. 3a). Flow cytometry analysis revealed that ObR expression was markedly increased in $\mathrm{PAH}$ patients, as compared with controls (figs $3 b$ and $c$ and table 2), either when expressed as percentage of Tregs (fig $3 c$ and table 2), or 

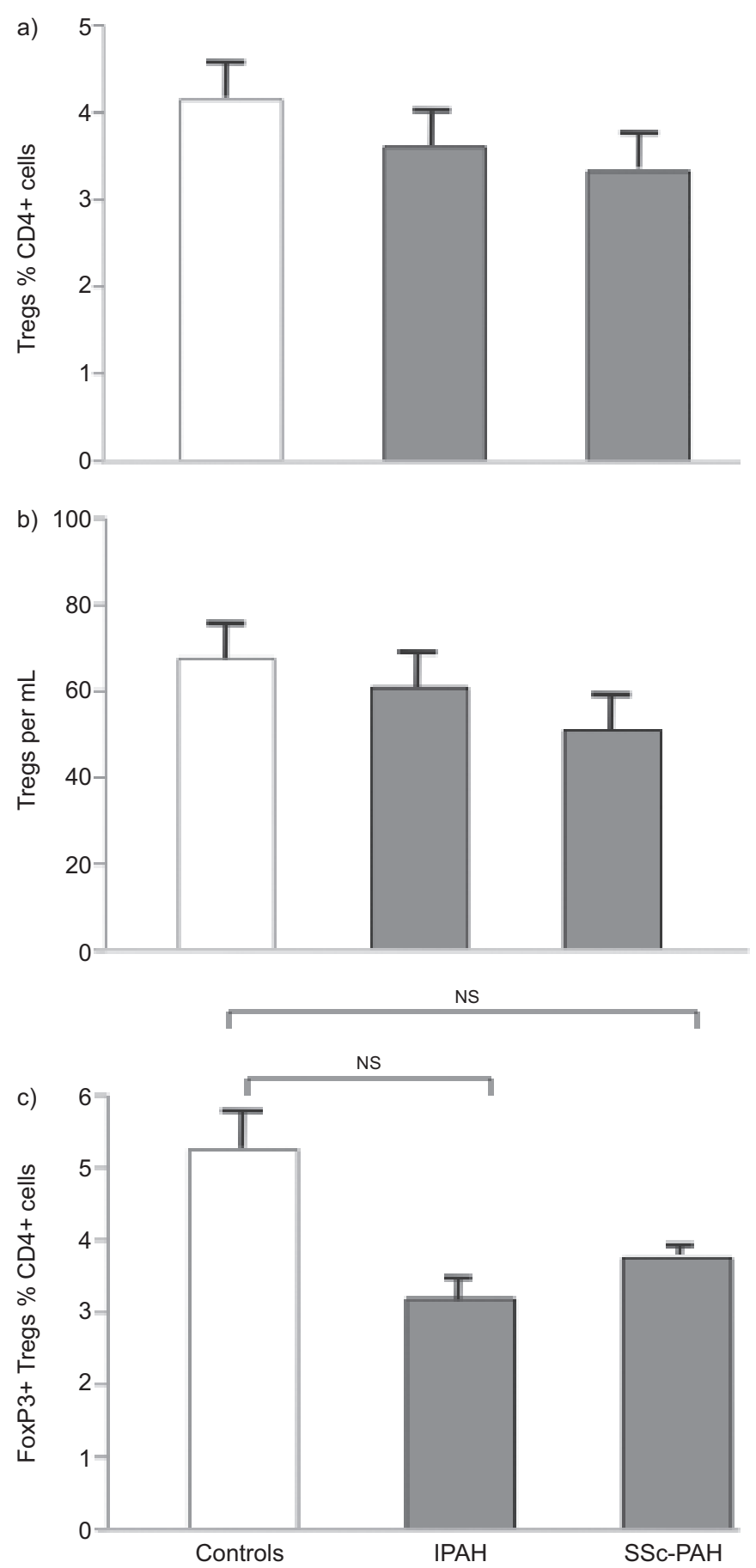

FIGURE 4. Regulatory T-lymphocytes (Tregs). a) Group data of circulating Tregs cell count, expressed as percentage of the total $\mathrm{CD} 4^{+}$population, in control subjects $(n=20)$, and in idiopathic pulmonary arterial hypertension (IPAH, $n=25)$ and scleroderma-associated pulmonary arterial hypertension (SSc-PAH, $\mathrm{n}=11$ ) patients. b) Group data of circulating Treg cell count, expressed as absolute numbers, in control subjects $(n=20)$, IPAH $(n=25)$ and SSc-PAH $(n=11)$ patients. c) Group data of circulating Treg cell count, defined as $\mathrm{CD} 4^{+} \mathrm{CD} 25^{+} \mathrm{CD} 127^{+} \mathrm{FoxP} 3^{+}$ and expressed as percentage of the total $\mathrm{CD} 4^{+}$population in control subjects $(n=5)$, IPAH $(n=5)$ and SSc-PAH $(n=5)$ patients. Data are presented as mean \pm SEM. NS: nonsignificant.

\section{TABLE 2 Regulatory T-lymphocytes (Tregs), leptin receptor and serum leptin data}

\begin{tabular}{|c|c|c|c|}
\hline & Controls & IPAH & SSc-PAH \\
\hline Treg $\%$ CD $4^{+}$cells & $4 \pm 0.5$ & $4 \pm 0.4$ & $3 \pm 0.4$ \\
\hline Treg per $\mathrm{mL}$ & $67 \pm 8$ & $61 \pm 8$ & $51 \pm 9$ \\
\hline FoxP3+ Treg $\% \mathrm{CD}^{+}$cells & $5 \pm 0.8$ & $3 \pm 0.4$ & $4 \pm 0.2$ \\
\hline ObR \% Treg & $16 \pm 5^{*}$ & $39 \pm 4$ & $36 \pm 9$ \\
\hline ObR per $\mathrm{mL}$ & $6 \pm 2^{*}$ & $19 \pm 2$ & $22 \pm 8$ \\
\hline ObR MFI & $378 \pm 46$ & $313 \pm 58$ & $466 \pm 21$ \\
\hline pSTAT3 \% ObR ${ }^{+}$Treg & $8 \pm 1.6^{*}$ & $4 \pm 0.5$ & $4 \pm 0.6$ \\
\hline PSTAT3 MFI & $1090 \pm 72$ & $1152 \pm 58$ & $1134 \pm 46$ \\
\hline Leptin $\mathbf{n g} \cdot \mathrm{mL}^{-1}$ & $2 \pm 0.3^{*}$ & $6 \pm 0.9$ & $6 \pm 1.4$ \\
\hline Circulatings $\mathrm{ObR} \mathbf{n g} \cdot \mathrm{mL}^{-1}$ & $27 \pm 1$ & $27 \pm 2$ & $22 \pm 2$ \\
\hline
\end{tabular}

Data are presented as mean \pm SEM. IPAH: idiopathic pulmonary arterial hypertension; SSc-PAH: scleroderma-associated pulmonary arterial hypertension; FoxP3: forkhead box P3; ObR: leptin receptor; MFI: mean fluorescence intensity; PSTAT3: phosphorylated signal transducer and activator of transcription 3; sObR: soluble leptin receptor. *: $p<0.05$ between controls versus IPAH and versus SSc-PAH patients.

as absolute numbers (fig $3 \mathrm{~d}$ and table 2). ObR expression was similar in IPAH and SSc-PAH. Interestingly, the expression of ObR on Tregs cell membranes did not correlate with BMI or serum leptin levels (figs $3 \mathrm{e}-\mathrm{h}$ ).

Taken together, these findings demonstrate that ObR expression is markedly increased in IPAH patients compared with controls and to a similar extent as in SSc-PAH. Interestingly, the level of ObR expression is independent of the patients' BMI or serum leptin levels.

\section{Circulating Treg levels}

To further explore the role of leptin on Tregs in PAH, we first examined the Treg cell count. Flow cytometry analysis revealed a normal Treg cell count in all groups. We expressed the Tregs as percentage of $\mathrm{CD}^{+}$cells as well as in absolute numbers and also found a normal Treg count (figs $4 a$ and $b$ and table 2). These data indicate that leptin does not seem to influence Treg numbers in PAH patients.

\section{Functional status of Tregs}

Our next objective was to determine the extent to which leptin could play a role on Treg function. As a readout of ObR activity, we measured pSTAT3 amounts, because STAT3 is known to participate in the intracellular signalling pathways of ObR [11]. After Treg permeabilisation, we quantified pSTAT3 by flow cytometry (fig. 5a). Interestingly, the number of pSTAT3 $^{+}$Tregs was markedly decreased in PAH patients compared with controls, and similarly in IPAH and SSc-PAH (figs $5 b$ and $c$ and table 2). In order to further investigate the relationship between leptin and Treg function, we stimulated freshly isolated PBMCs from healthy controls with recombinant leptin, we stained them as described above and measured pSTAT3 by flow cytometry. pSTAT3 ${ }^{+}$Tregs were significantly decreased when stimulated by leptin, clearly showing a leptindependent effect on Treg functional status (fig. 5d). Taking 
a)
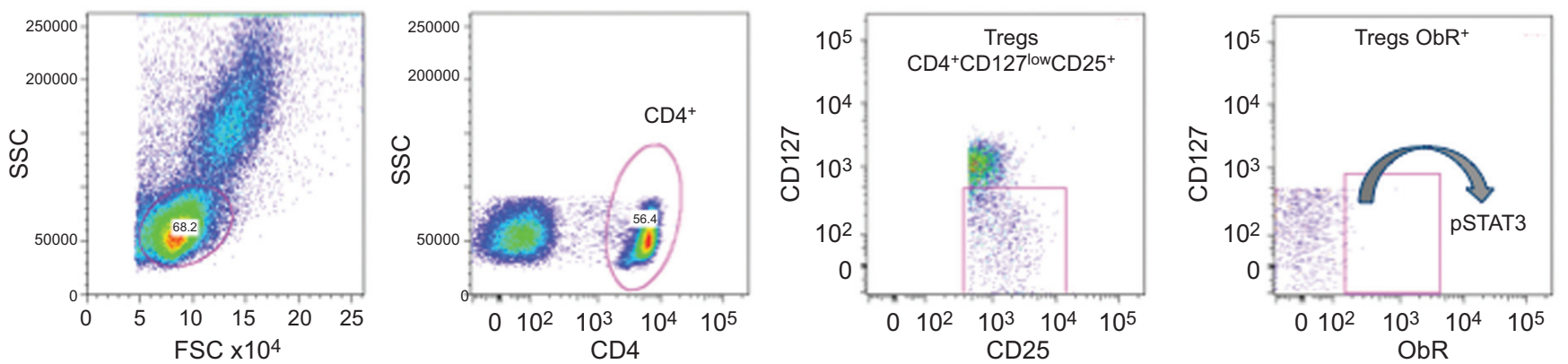

b)
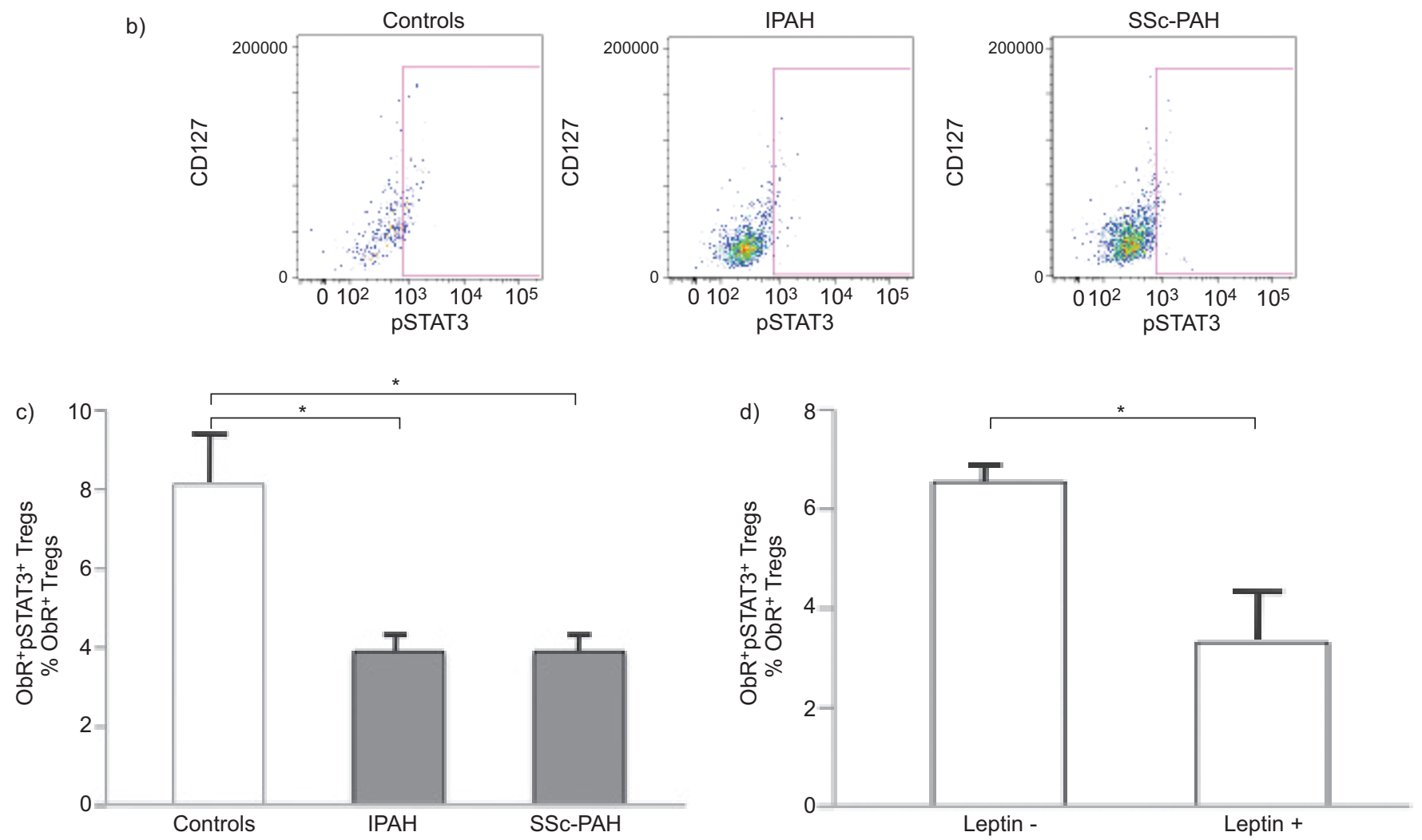

FIGURE 5. Functional status of regulatory T-lymphocytes (Tregs) expressing leptin receptor (ObR). a) Single fluorescence-activated cell sorter (FACS) dot plots representing an example of staining strategy by using surface markers to phenotype Tregs and intracellular staining with anti-phosphorylated signal transducer and activator of transcription 3 (pSTAT3) antibody, analysed by flow cytometry in a control subject. b) Single FACS dot plot representing pSTAT3 in ObR ${ }^{+}$Tregs in control subjects, idiopathic pulmonary arterial hypertension (IPAH) and scleroderma-associated pulmonary arterial hypertension (SSc-PAH) patients. SSC: side scatter; FSC: forward scatter. c) Group data of $\mathrm{ObR}^{+}$pSTAT3 $^{+}$Tregs, expressed as percentage of the total $\mathrm{ObR}^{+}$Treg population, in control subjects $(n=20)$, and IPAH ( $\left.n=25\right)$ and SSc-PAH ( $n=11$ ) patients. *: $p<0.05$ between controls versus IPAH and versus SSc-PAH patients. d) Group data of $\mathrm{ObR}^{+}$pSTAT3 $^{+}$Tregs, expressed as percentage of the total ObR ${ }^{+}$Treg population in control subjects peripheral blood mononuclear cells $(n=3)$ after stimulation with or without leptin. *: $p<0.05 . c)$ and d) Data are presented as mean \pm SEM.

these results together, we hypothesise that leptin regulates Tregs in PAH by inhibiting them through ObR binding.

\section{Leptin secretion by endothelial cells}

Leptin has recently been detected in lung tissue [20]. As our findings suggested a mechanistic role for leptin in Treg functional regulation and P-ECs represent a critical cell type in IPAH patients [16], our next aim was to investigate whether PECs could represent one of the sources of leptin in PAH. To test this hypothesis, after exclusion of inflammatory mechanisms as a source of leptin (figs $2 b$ and c), we performed immunohistochemistry on lung specimens from controls and IPAH patients, and we stained them for leptin and ObR. A more intense immunoreactivity was noted for leptin in the endothelium of distal pulmonary arterial walls in IPAH patients versus controls. In contrast, no significant changes in $\mathrm{ObR}$ were found between IPAH patients and control. Therefore, we assessed that P-ECs are an important source of leptin in the lung. To further assess whether the synthesis and the release of leptin by P-ECs were increased in $\mathrm{PAH}$, we isolated microvascular P-EC from lung specimens from IPAH patients and from controls. We measured the amount of leptin protein in the cell supernatant and found a significant increase in leptin in IPAH patients compared with controls (fig. 6). These findings indicate that 

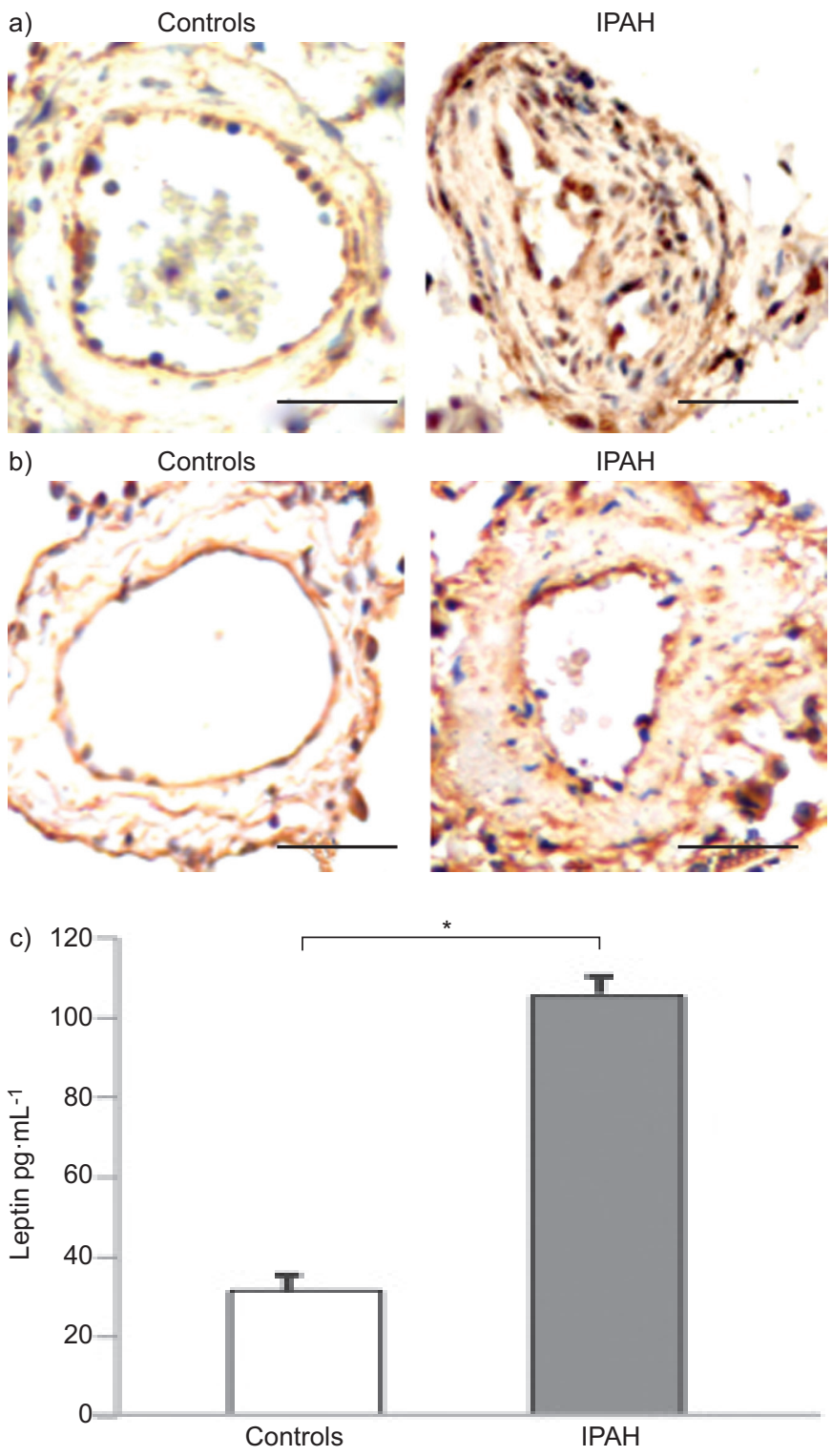

FIGURE 6. Leptin production by human pulmonary endothelial cells. a) Staining for leptin in lung specimens in a representative pulmonary artery from controls and idiopathic pulmonary arterial hypertension (IPAH) patients. b) Staining for leptin receptor in lung specimens in a representative pulmonary artery from controls and IPAH patients. Scale bars $=50 \mu \mathrm{m}$. c) Group data of leptin production by human pulmonary endothelial cells, in control subjects $(n=15)$ and in IPAH patients $(n=10)$. Data are presented as mean \pm SEM. ${ }^{*}: p<0.05$ between controls versus IPAH patients.

P-ECs contribute to the increased secretion of leptin measured in IPAH patients.

\section{DISCUSSION}

One of the central unanswered questions in $\mathrm{PAH}$ pathogenesis relates to the role of inflammation and dysimmunity in the development and/or progression of IPAH. It remains unclear how immune mechanisms contribute to the pathogenesis of IPAH. We approached this question by determining the role of leptin and endothelial dysfunction in circulating Treg regulation. Our data suggest, to the best of our knowledge, for the first time, that leptin may play a role in the immunopathogenesis of IPAH by inhibiting Treg function through its receptor upregulation on circulating Treg surfaces. Furthermore, we also show, using P-ECs isolated from PAH patients and lung tissues, that P-ECs could represent one of the sources of leptin.

\section{Leptin, Treg function and ObR expression upregulation}

Despite strong evidence for the role of leptin in autoimmunity, the precise mechanism of its activity is still controversial. Much of the difficulty relies on the highly pleiotropic activities of leptin on both the neuroendocrine and immune systems. In the immune system, leptin can directly affect the activity of numerous immune cell types of both the innate and the adaptive systems. A critical protective role for Tregs in several autoimmune diseases is now well established [14, 15, 17], but it is still controversial whether leptin induces Treg anergy and/or hyporesponsiveness in autoimmune diseases. In order to identify Tregs by flow cytometry, we have chosen to use surface markers because recent studies have indicated that, in humans, expression of endogenous forkhead box P3 (FoxP3) is not sufficient to induce Treg activity or to identify Tregs [21, 22].

In this study, we have established a unique link between leptin and Tregs in PAH by showing that leptin can modulate the hyporesponsiveness of Tregs in vivo. Freshly isolated Tregs from $\mathrm{PAH}$ patients display higher expression of ObR on their surface compared with healthy controls. At the molecular level, circulating PAH Tregs express low levels of pSTAT3, which represents the major signalling pathway in Tregs. In contrast, the number of circulating Tregs is in the normal range and it is similar in $\mathrm{PAH}$ patients and controls. Interestingly, these results were similar in the two major PAH subgroups tested, IPAH and $\mathrm{SSc}-\mathrm{PAH}$, in which there is an established associated immunological disorder. Furthermore, when stimulated by recombinant leptin, Tregs express lower levels of pSTAT3, clearly indicating a direct effect of leptin on Treg functional status. It appears that, in PAH, leptin controls Treg function rather than controlling the numbers of Tregs that could participate in the immunopathogenesis of PAH.

\section{Leptin and inflammation}

Leptin production is not only regulated by food intake, but also by various hormones, as well as by several inflammatory mediators, both in humans and in experimental models [10, 11]. The pro-inflammatory properties of leptin are similar to those of other acute-phase reactants. Generally, leptin increases during the course of acute infection, sepsis and inflammation [10,11]. It is now clear that inflammation plays an important role in $\mathrm{PAH}$ pathogenesis. Conversely, some studies have suggested an association between leptin levels and inflammatory markers, such as soluble TNF receptors or CRP [23]; this has not been confirmed by others [24]. We approached this controversial question by measuring serum pro-inflammatory cytokine levels in PAH and controls. Conversely to recent data published by QUARCK et al. [25], we found normal levels of CRP without any difference within the groups. We confirmed our previous data showing an increase in IL- 6 and $-1 \beta$, and normal levels of TNF- $\alpha$ in PAH patients [2]. We showed no correlation between leptin levels and inflammatory markers. It is noteworthy that patients did not receive therapies susceptible to decrease inflammatory markers, such as statins, and there was no active systemic 
disease in the patients with SSc-PAH that we studied, as shown by the lack of steroid and/or immunosuppressant therapy at the time of SSc-PAH management, and the normal CRP levels measured in these subjects. This suggests that in the PAH patients we studied, increased levels of leptin were not linked to markers of systemic inflammation.

\section{P-ECs as a source of leptin?}

Leptin expression and secretion are constitutive in adipocytes and, at first, were thought to be restricted only to this tissue; however, recent reports have demonstrated that leptin is also produced at low levels by other tissues [26]. Interestingly, it has been shown that another important source of leptin is the Tregs themselves, which both secrete leptin and express leptin receptor [10]. Thus, leptin can mediate a negative autocrine loop in Tregs, which can promote the onset and/or the progression of autoimmune diseases [22]. Theoretically, the blockade of leptin by antagonists, antibodies or soluble receptors should inhibit leptin bioavailability and reduce its effects. So far, this has been shown in animal models with experimental conditions [10]. In humans, leptin administration does not efficiently improve immune function in the normal or obese individual but only in individuals with congenital leptin deficiency and lipodystrophy [27]. The question of the existence of another source of leptin involved in the immune regulation in humans remains unanswered. Endothelial dysfunction represents one the major cellular PAH characteristics that could contribute to increased leptin secretion [3]. By using P-ECs isolated from $\mathrm{PAH}$ and lung specimens, we show here, for the first time, that PAH pulmonary endothelial cells produce more leptin than controls. This can explain, at least in part, the increased serum leptin levels in PAH patients compared with controls, despite the normal number of Tregs. Further studies are needed to confirm that endothelial cells represent the source of leptin and the role of leptin in Tregs in PAH.

\section{Conclusions}

In conclusion, our findings address a central unanswered question in $\mathrm{PAH}$ pathogenesis, namely that relating to the role of dysimmunity in the development and/or progression of IPAH. Our evidence that leptin and endothelial cells play a determining role in the regulation of Tregs in IPAH reveals a previously unknown mechanism in IPAH immunopathogenesis that can contribute to the onset and/or progression of the disease. Our findings indicate for the first time that leptin must be considered as a mediator of immunological disorders in IPAH.

\section{SUPPORT STATEMENT}

A. Huertas is supported by the Josso Award 2010 from the French Medical Research Foundation. B. Girerd is funded by the Assistance Publique-Hôpitaux de Paris (PHRC NCT01600898).

\section{STATEMENT OF INTEREST}

Statements of interest for D. Montani and M. Humbert can be found at www.erj.ersjournals.com/site/misc/statements.xhtml

\section{REFERENCES}

1 Simonneau G, Robbin IM, Beghetti M, et al. Updated clinical classification of pulmonary hypertension. J Am Coll Cardiol 2009; 54: Suppl. 1, S43-S54.
2 Humbert M, Monti G, Brenot F, et al. Increased interleukin-1 and interleukin-6 serum concentrations in severe primary pulmonary hypertension. Am J Respir Crit Care Med 1995; 151: 1628-1631.

3 Humbert M, Morrell NW, Archer SL, et al. Cellular and molecular pathobiology of pulmonary arterial hypertension. J Am Coll Cardiol 2004; 43: Suppl. 12, 13S-24S.

4 Perros F, Dorfmüller P, Souza R, et al. Dendritic cell recruitment in lesions of human and experimental pulmonary hypertension. Eur Respir J 2007; 29: 462-468.

5 Dorfmuller P, Perros F, Balabanian K, et al. Inflammation in pulmonary arterial hypertension. Eur Respir J 2003; 22: 358-363.

6 Tuder RM, Groves B, Badesch DB, et al. Exuberant endothelial cell growth and elements of inflammation are present in plexiform lesions of pulmonary hypertension. Am J Pathol 1994; 144: 275-285.

7 Tamby MC, Chanseaud Y, Humbert M, et al. Anti-endothelial cell antibodies in idiopathic and systemic sclerosis associated pulmonary arterial hypertension. Thorax 2005; 60: 765-772.

8 Terrier B, Tamby MC, Camoin L, et al. Identification of target antigens of antifibroblast antibodies in pulmonary arterial hypertension. Am J Respir Crit Care Med 2008; 177: 1128-1133.

9 Tamosiuniene R, Tian W, Dhillon G, et al. Regulatory T cells limit vascular endothelial injury and prevent pulmonary hypertension. Circ Res 2011; 109: 867-879.

10 Lam QL, Lu L. Role of leptin in immunity. Cell Mol Immunol 2007; 4: 1-13.

11 La Cava A, Matarese G. The weight of leptin in immunity. Nat Rev Immunol 2004; 4: 371-379.

12 Lord GM, Matarese G, Howard JK, et al. Leptin modulates the Tcell immune response and reverses starvation-induced immunosuppression. Nature 1998; 394: 897-901.

13 Lam QL, Liu S, Cao X, et al. Involvement of leptin signaling in the survival and maturation of bone marrow-derived dendritic cells. Eur J Immunol 2006; 36: 3118-3130.

14 Matarese G, Carrieri PB, La Cava A, et al. Leptin increase in multiple sclerosis associates with reduced number of CD4(1)CD251 regulatory T cells. Proc Natl Acad Sci USA 2005; 102: 5150-5155.

15 Wing K, Sakaguchi S. Regulatory T cells exert checks and balances on self tolerance and autoimmunity. Nat Immunol 2010; 11: 7-13.

16 Cool CD, Stewart JS, Wehareha P, et al. Three-dimensional reconstruction of pulmonary arteries in plexiform pulmonary hypertension using cell-specific markers. Evidence for a dynamic and heterogeneous process of pulmonary endothelial cell growth. Am J Pathol 1999; 155: 411-419.

17 Liu W, Putnam AL, Xu-Yu Z, et al. CD127 expression inversely correlates with FoxP3 and suppressive function of human CD4+ T reg cells. J Exp Med 2006; 203: 1701-1711.

18 De Rosa V, Procaccini C, Calì G, et al. A key role of leptin in the control of regulatory $\mathrm{T}$ cell proliferation. Immunity 2007; 26: 241-255.

$19 \mathrm{Tu} \mathrm{L}$, Dewachter L, Gore B, et al. Autocrine FGF2 signaling contributes to altered endothelial phenotype in pulmonary hypertension. Am J Respir Cell Mol Biol 2011; 45: 311-322.

20 Bellmeyer A, Martino JM, Chandel NS, et al. Leptin resistance protects mice from hyperoxia-induced acute lung injury. Am J Respir Crit Care Med 2007; 175: 587-594.

21 Gavin MA, Torgerson TR, Houston E, et al. Single-cell analysis of normal and FOXP3-mutant human T cells: FOXP3 expression without regulatory T cell development. Proc Natl Acad Sci USA 2006; 103: 6659-6664.

22 Wang J, Ioan-Facsinay A, van der Voort EI, et al. Transient expression of FOXP3 in human activated nonregulatory CD4+ T cells. Eur J Immunol 2007; 37: 129-138.

23 Shamsuzzaman AS, Winnicki M, Wolk R, et al. Independent association between plasma leptin and C-reactive protein in healthy humans. Circulation 2004; 109: 2181-2185. 
24 Gomez-Ambrosi J, Salvador J, Silva C, et al. Leptin therapy does not affect inflammatory markers. J Clin Endocrinol Metab 2005; 90: 3803.

25 Quarck R, Nawrot T, Meyns B, et al. C-reactive protein: a new predictor of adverse outcome in pulmonary arterial hypertension. J Am Coll Cardiol 2009; 53: 1211-1218.
26 Flier JS. Obesity wars: molecular progress confronts an expanding epidemic. Cell 2004; 116: 337-350.

27 Farooqi IS, Matarese G, Lord GM, et al. Beneficial effects of leptin on obesity, $\mathrm{T}$ cell hyporesponsiveness, and neuroendocrine/ metabolic dysfunction of human congenital leptin deficiency. J Clin Invest 2002; 110: 1093-1103. 